\title{
Dental functional status with and without tooth replacement in a Chinese adult population
}

\author{
Qian Zhang • Dick J. Witter • Ewald M. Bronkhorst • \\ Muyun Jia • Nico H. J. Creugers
}

Received: 25 January 2011 / Accepted: 7 September 2011 /Published online: 29 September 2011

(C) The Author(s) 2011. This article is published with open access at Springerlink.com

\begin{abstract}
The objective of this study is to investigate the prevalence of missing teeth and prosthodontic replacements in a Chinese adult population using a hierarchical dental functional classification system. A total of 1,462 dentate subjects over 40 years from Shandong Province, China were included and categorized in the functional classification system with and without tooth replacements. Depending on replacements, subjects could be reclassified
\end{abstract}

\section{Q. Zhang $(\bowtie)$}

Department of Prosthetic Dentistry,

Affiliated Hospital of Medical School, Qingdao University,

Jiangsu Road 16\#,

Qingdao, People's Republic of China

e-mail: habebeq@hotmail.com

D. J. Witter · N. H. J. Creugers

Department of Oral Function and Prosthetic Dentistry, College of Dental Science, Radboud University Nijmegen Medical Centre, Philips van Leydenlaan 25,

6525 EX Nijmegen, The Netherlands

D. J. Witter

e-mail: d.witter@dent.umcn.nl

N. H. J. Creugers

e-mail: n.creugers@dent.umcn.nl

E. M. Bronkhorst

Department of Preventive and Restorative Dentistry, College of Dental Science, Radboud University Nijmegen Medical Centre, Philips van Leydenlaan 25,

6525 EX Nijmegen, The Netherlands

e-mail: e.bronkhorst@dent.umcn.nl

\section{Jia}

Department of Oral and Maxillofacial Surgery,

Affiliated Hospital of Medical School, Qingdao University, Jiangsu Road 16\#,

Qingdao, People's Republic of China

e-mail: jiamuyun@gmail.com (promoted) to categories reflecting higher functionality. "Promotions" were considered indicators for prosthodontic effectiveness. Homogeneities after dichotomization into functional categories appeared to be moderate to good. In the " $\geq 10$ teeth in each jaw" branch, mean number of teeth and posterior occluding pairs were $27.93 \pm 2.74$ and $7.10 \pm$ 1.94 , respectively. In the branch " $<10$ teeth in each jaw," these figures were $16.17 \pm 5.54$ and $1.49 \pm 1.45$. Fixed dental prostheses (FDPs) added on average 3.5 artificial teeth; $46 \%$ of subjects with FDP promoted to a higher functional level. For removable dental prostheses (RDPs), these numbers were $8.5 \%$ and $79 \%$, respectively. Promotion value per tooth added was significantly higher for FDPs. The classification system was able to quantify the effectiveness of teeth replacements. It was shown that RDPs were more effective when higher numbers of teeth were replaced, while FDPs were more effective per artificial tooth added.

Keywords Tooth loss · Tooth replacement · Hierarchical dental functional classification · Posterior occluding pairs

\section{Introduction}

Tooth loss among adults is a common phenomenon in China. Several oral epidemiological surveys concluded that missing teeth contribute most to the relatively high decayed/missing/filled teeth (DMFT) scores reported for Chinese adults $[1,2]$. The number of "20 natural teeth" is the generally accepted WHO operative criterion for a functional natural dentition [3]. Results of a systematic review based on DMFT data showed that Chinese subjects of 40 years have on average 26 teeth present and an average of 20 teeth present at the age of 65 years [4]. Unfortunately, 
the review was unable to detect prosthodontic tooth replacements and its potential influence on functionality.

Although many epidemiological studies express oral functionality by numbers of teeth, it is questioned whether just the number of teeth is adequate to describe the functional status of dentitions. It has been claimed that occluding pairs of natural teeth are strongly correlated with oral functional impacts [5]. A recent systematic review provided circumstantial evidence that beside the number of teeth, also tooth type, tooth location, and number of occluding pairs determine oral functionality [6]. Several authors have made attempts to classify dentitions according to these variables. Recently, the Eichner index has been validated for an elderly Japanese population with respect to masticatory function [7]. However, the Eichner index does not specify the number and location of teeth present and is therefore not reflecting oral functionality in detail, for example aesthetics. A hierarchical classification system that reflects oral functionality was developed by our group and includes the number of natural teeth, their location in terms of dental region, and the number of posterior occluding pairs of natural teeth (POPs). The system enables the mapping of the large variety in configurations of dentitions and has been validated for an adult Vietnamese population with respect to chewing ability [8].

A Japanese study demonstrated that chewing ability significantly correlates with the number of natural teeth. However, if the total number of functional tooth units increases as a result of removable dental prostheses (RDPs), masticatory function did not yield a significant improvement [9]. Fixed dental prostheses (FDPs), however, are thought to provide similar functionality as natural teeth $[9$, 10]. For this reason, RDPs and FDPs in cases of upgrading dentitions to higher levels of functionality have to be distinguished.

It was the purpose of the present study to map the dental status, including the effects of tooth replacements, in a Chinese adult population by means of the "Nijmegen" hierarchical dental functional classification system. It was hypothesized that effectiveness of prosthodontic replacement can be quantified by the hierarchical classification system.

\section{Materials and methods}

\section{Sampling construction}

The study was conducted in the Qingdao area, located at the east coast of Shandong Province, the latter situated in Eastern China. Shandong is one of the largest provinces in China in terms of population (94 million in 2008) and economy. Qingdao City has approximately 3 million inhabitants. Qingdao City has direct jurisdiction over the surrounding rural territory in Shandong Province, including five county-level cities (approximately 200,000-400,000 citizens each) and the counties surrounding these cities. Each county comprises $40-80$ small rural villages. The total area of Qingdao (urban and rural) is approximately $10,000 \mathrm{~km}^{2}$ with a coastline of approximately $730 \mathrm{~km}$ and has approximately 8 million inhabitants.

For this study, a cross-sectional survey, representing 1,588 subjects aged $\geq 40$ years living in urban and rural areas in Qingdao, Shandong Province, was conducted (Table 1). Subjects were selected randomly from administrative lists of residents of communities or villages provided by local authorities and lists of employees of factories. Inclusion aimed at proportional distribution according to age, gender, and residence (urban or rural). In cases where subjects were invited but did not show up, other subjects were randomly drawn from the same sampling lists. Data were collected in 2009 and 2010.

The urban sample was constructed after consulting local authorities on the basis of accessibility and comprised 11 communities and four factories in Qingdao City. Administrators of the communities informed and invited their residents for participation in this study. The examination venue usually was a neighborhood community office or a social center for elderly. A total of 570 community inhabitants and 193 employees from factories were included. As it appeared that subjects of certain age groups were underrepresented in the initial urban sample (mostly male), a complementary convenient sub-sample was eventually included. Subjects from name lists of a health care center affiliated to a general hospital attending for periodic checkup were invited to participate. In this way, 53 subjects were included.

For the rural sample, one county (Zhugou) considered representative for northeast Shandong Province was chosen on the basis of accessibility. This county (total population approximately 36,000 ) is located approximately $120 \mathrm{~km}$ northwest from Qingdao City and comprises 56 villages ranging from 153 to 1,583 inhabitants. Of these villages, ten were selected randomly: three villages out of 19 with highest 2008 Gross Domestic Product (GDP), four out of 18 with middle GDP, and three out of 19 with lowest GDP.

Table 1 Number (percent) of included subjects according to gender and residence

\begin{tabular}{lllc}
\hline & Urban & Rural & Total \\
\hline Female & $419(54)$ & $350(46)$ & $769(48)$ \\
Male & $396(48)$ & $423(52)$ & $819(52)$ \\
Total & $815(51)$ & $773(49)$ & $1588(100)$ \\
\hline
\end{tabular}


Subjects were randomly selected using administrative name lists.

\section{Clinical examination}

After obtaining verbal consent from the participants, a clinical examination was conducted by a calibrated examiner following the procedures and diagnostic criteria recommended by the World Health Organization [11]. Of all variables recorded, only the presence of teeth (including third molars), tooth type, number and location of POPs, and tooth replacements were considered in the present study. Inter-observer agreements between the principal investigator and experienced researchers in the field were excellent (kappa's $\geq 0.89$ ). A tooth root was defined as missing. A replaced tooth was defined as a missing tooth replaced by a FDP or RDP. The research was carried out in compliance with the Helsinki Declaration and was approved by the ethics committee of the medical school at Qingdao University, Qingdao, China.

\section{Hierarchical dental functional classification system}

Dentitions were classified on the basis of a dichotomized five level branching hierarchy in which the criteria applied on the levels are based on conditions that reflect functionality (Table 2). This classification system has been used in an epidemiological study on oral function in Vietnam and has been described previously [8]. The conditions used are the number of natural teeth, the tooth types present, and the number of POPs. For practical reasons, categories with relatively low prevalence (in the "at least ten teeth branch," the categories not meeting the cut-offs, in the "less than ten teeth branch," the categories that met the cut-offs) were not further dichotomized to the next level.

Data analyses

For the classification of dental configurations, only natural teeth were considered. Subjects being edentulous in one or both jaws were excluded from the analyses. With regard to each level in the branching hierarchy, percentages of subjects meeting or not meeting the classification criteria were calculated. Mean numbers of teeth present and mean numbers of POPs were calculated for four age groups: (1) 40-49 years, (2) 50-59 years, (3) 60-69 years, and (4) over 70 years.

Intraclass correlation coefficients (ICCs) were calculated as a measure for the homogeneity of the groups after dichotomization at each level with respect to the number of teeth (ICC-t) and the number of POPs (ICC-p). The homogeneity of groups or categories after dichotomization is considered a measure that reflects the significance of the cut-off and the validity of the classification system. A bootstrap procedure with 1,000 resamplings was used to determine the standard errors (SE) for the respective ICCs.

In the next step, also replacement of teeth was considered. For all categories in the functional classification system described above, the mean number of replaced teeth was calculated per age group and added to the mean number of natural teeth. A distinction was made between teeth replaced by FDP and those replaced by RDP. In addition, mean number of POPs added by FDPs (F-POPs) and RDPs (R-POPs) were calculated for each category in the functional classification system per age group.

If, on the basis of teeth added by FDP or RDP, a subject could be reclassified to a category reflecting higher functionality, i.e., from not meeting the level-specific criterion to meeting the particular criterion (see Table 2), this was considered to be a "promotion."

These "promotions" were considered as indicators for prosthodontic effectiveness in terms of added value in the dental functional status. The value 1 was given for a promotion to category "sufficient molar region," the value 2 for a promotion to category "sufficient premolar region," promotion to "complete anterior region" was given the value 3 , and promotion to category "at least ten teeth in each jaw" was given the value 4 .

When subjects were reclassified to more than one higher level in the functional classification system, the values were

Table 2 Levels and criteria for dichotomization of the step-by-step branching hierarchy used

\begin{tabular}{llll}
\hline Level & \multicolumn{2}{l}{ Meeting criterion } & Dichotomy \\
\cline { 2 - 4 } & Yes & No & $\geq 1$ tooth vs. no teeth \\
\hline I Dentition level & $\geq 1$ tooth present in each jaw & Edentulous jaw(s) & $\geq 10$ teeth vs. $<10$ teeth \\
II Jaw level & $\geq 10$ teeth in both upper and lower jaw & $<10$ teeth in upper or lower jaw & Complete vs. incomplete \\
III Anterior level & All 12 anterior teeth present & $<12$ anterior teeth & $\leq 2$ occluding pairs of premolars \\
IV Premolar level & 3 or 4 occluding pairs of premolars & "Sufficient" vs. "impaired" \\
V Molar level & $\geq 1$ occluding pairs of molars at both left & No occluding pairs of molars at left & "Sufficient" vs. "impaired" \\
& and right side of the dentition & &
\end{tabular}


summed (e.g., from category "insufficient molar region" to "sufficient molar region" (value=1 point) and "sufficient premolar region" (value $=2$ points) by restoring an impaired molar region and an impaired premolar region with tooth replacements, the values were summed to total score $=3$ points). Some examples of promotions are displayed in Fig. 1.

It was hypothesized that effectiveness of prosthodontic replacement in terms of oral functionality can be quantified by promotions to higher levels in the hierarchical classification system. Secondly, as RDPs in general replace higher numbers of missing teeth compared to FDPs, it was also hypothesized that they are more effective in terms of upgrading the dental functional status.

\section{Results}

Natural teeth present and POPs

Of the total sample initially included in the study $(n=1,588)$, 126 edentulous subjects (8\%) in upper and/or lower jaw were excluded. The remaining 1,462 dentate subjects ( $92 \%)$ were included in the analyses.

In general, the classification of subjects in the hierarchical functional system resulted in varying homogeneity of the groups with respect to number of natural teeth present and POPs (Fig. 2). Highest ICCs, indicating good group homogeneity, were found at level II (ten teeth in each jaw): $0.857 \pm 0.008$ for the number of teeth (ICC-t) and $0.793 \pm$ 0.013 for number of POPs (ICC-p). However, low group homogeneities were found at the "anterior region complete" level (level III) in the branch "at least 10 teeth in each jaw" (ICC- $\mathrm{t}=0.295 \pm 0.038$ and ICC- $\mathrm{p}=0.017 \pm 0.015$ ).

The branching hierarchy describes $82 \%$ of all subjects with teeth in both jaws up to level IV (premolar region) and $73 \%$ up to level V (molar region). Forty-eight percent of the total sample met all criteria for a "sufficient" functional dentition (meeting all cut-offs up to level V); $10 \%$ met none of the criteria (apart from the $8 \%$ edentulous subjects).

Of the subjects with at least ten teeth in each jaw (76\% of the total sample), $82 \%$ ( $62 \%$ of the total sample) had an intact anterior region, $73 \%$ (55\% of the total sample) met the criterion for a "sufficient" premolar region, and $63 \%$ ( $48 \%$ of the total sample) had a "sufficient" molar region (Fig. 2). Overall, in the "at least ten teeth in each jaw" branch, mean number of teeth was $27.93 \pm 2.74$; mean number of POPs was $7.10 \pm 1.94$. In the branch of "less than ten teeth in each jaw," these figures were $16.17 \pm 5.54$ and $1.49 \pm 1.45$, respectively.

Subjects meeting the criterion "at least ten teeth in each jaw" had a mean number of $28.58 \pm 2.50$ teeth present and

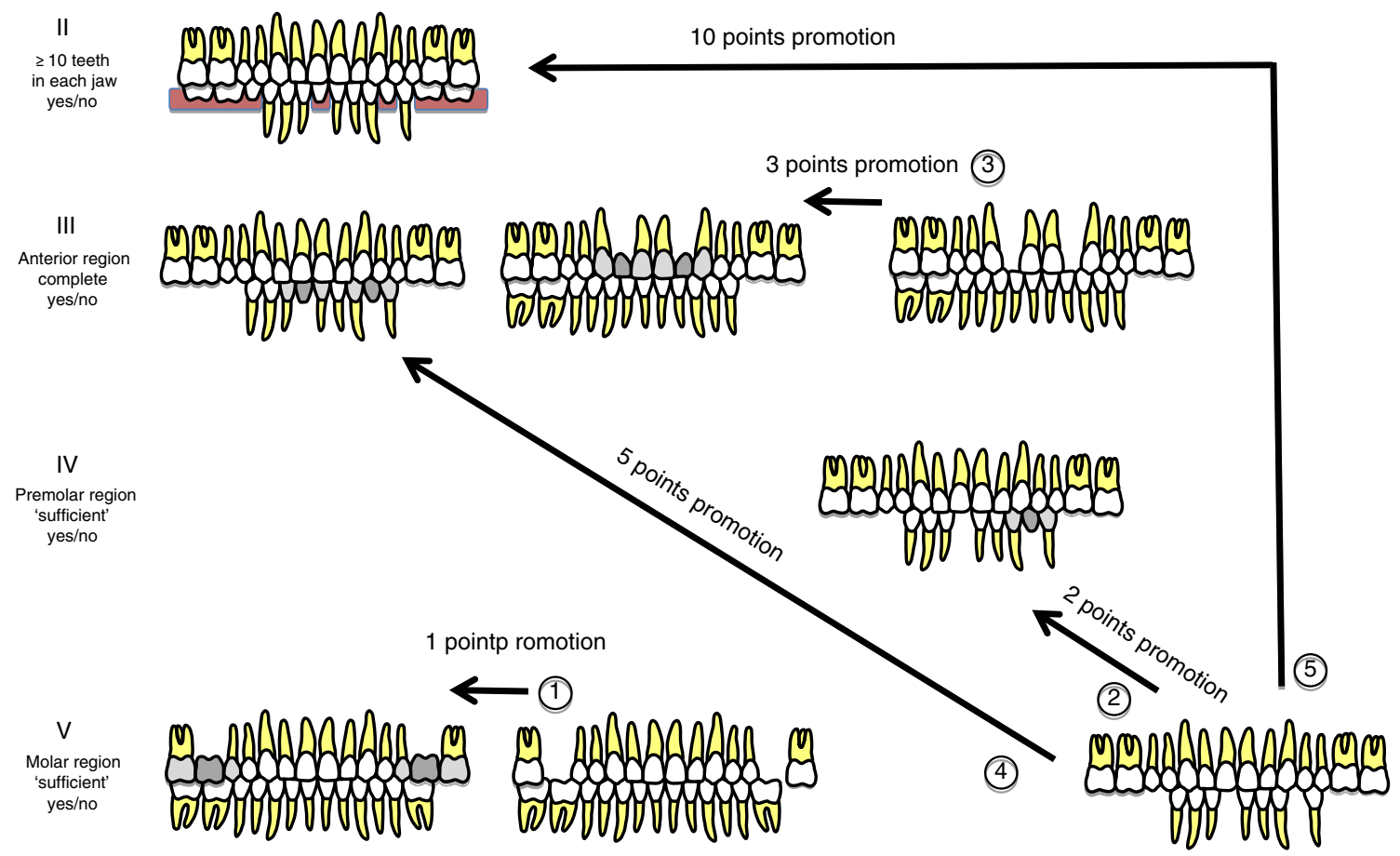

Fig. 1 Examples of promotions (reclassifications to categories reflecting higher functionality) by fixed and removable tooth replaments: (1) promotion to sufficient molar region by two FDPs (1 point); (2) promotion to sufficient premolar region by one FDP (2 points); (3) promotion to complete anterior region by two FDPs; (4) promotion to sufficient premolar region (2 point) and complete anterior region (3 points) by two FDPs; (5) promotion to sufficient molar (1 point) and premolar regions ( 2 points), complete anterior region ( 3 points) and more than ten teeth in each jaw (4 points) 


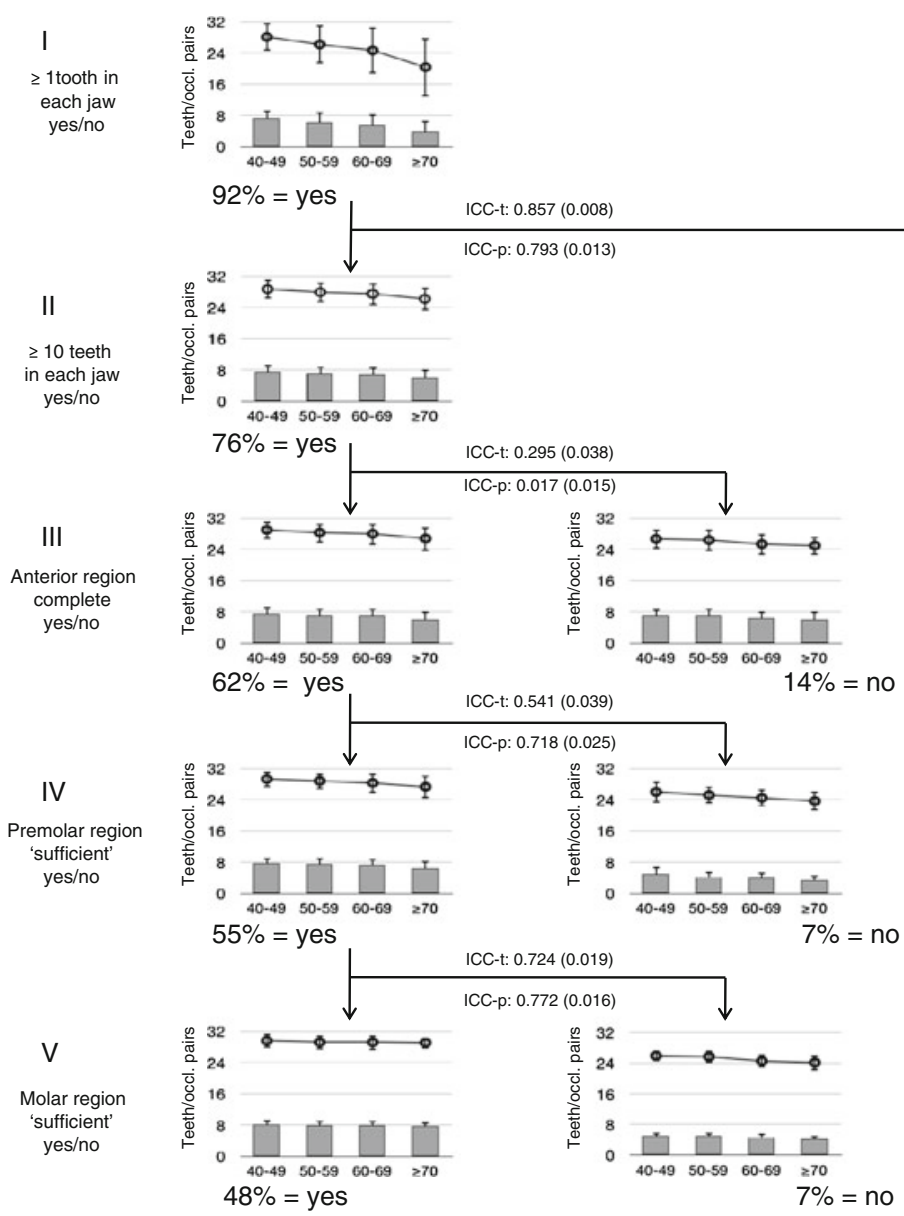

- Mean number of teeth present (vertical lines indicate SD)
ICC-t Intraclass correlation coefficient for number of natural teeth (SD)

ICC-p Intraclass correlation coefficient for number of natural posterior occluding pairs (POPs)
Fig. 2 Percentage of subjects and number of natural teeth and natural posterior occluding pairs per age group, according to the step-by-step branching hierarchy dichotomized (yes/no) at five levels: $I \geq 1$ tooth in each jaw, $I I \geq 10$ natural teeth in each jaw, $I I I$ anterior region complete, $I V$ premolar region "sufficient," $V$ molar region "sufficient"

branch of "at least ten teeth in each jaw," the average number of teeth replaced was relatively low (approximately 0.7 teeth, of which $82 \%$ were replaced by FDP). In that branch, the highest number of teeth replaced was found in the category with an incomplete anterior region, whereas the category "impaired premolar region" received the highest mean number of added POPs (F-POPs, 1.04; R-POPs, 0.27).

The vast majority of teeth replaced were found in the branch "less than ten teeth in each jaw": The average number

Table 3 Number of subjects (percent) with partial dental prostheses according to number of teeth replaced

\begin{tabular}{llll}
\hline $\begin{array}{l}\text { Number } \\
\text { of teeth } \\
\text { replaced }\end{array}$ & $\begin{array}{l}\text { Subjects } \\
\text { having } \\
\text { FDP }\end{array}$ & $\begin{array}{l}\text { Subjects } \\
\text { having } \\
\text { RDP }\end{array}$ & $\begin{array}{l}\text { Subjects having } \\
\text { FDP or RDP } \\
\text { or both }\end{array}$ \\
\hline $1-2$ & $251(57)$ & $36(22)$ & $262(47)$ \\
$3-7$ & $169(38)$ & $57(36)$ & $204(37)$ \\
$>7$ & $21(5)$ & $67(42)$ & $92(16)$ \\
Total & $441(100)$ & $160(100)$ & $558(100)$ \\
\hline
\end{tabular}




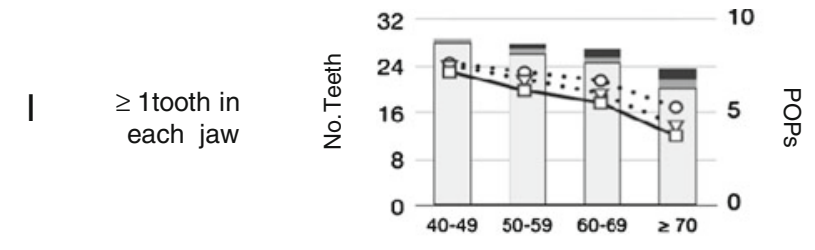

SDs T: $2.62-7.55$ SDs P: $1.87-3.01$

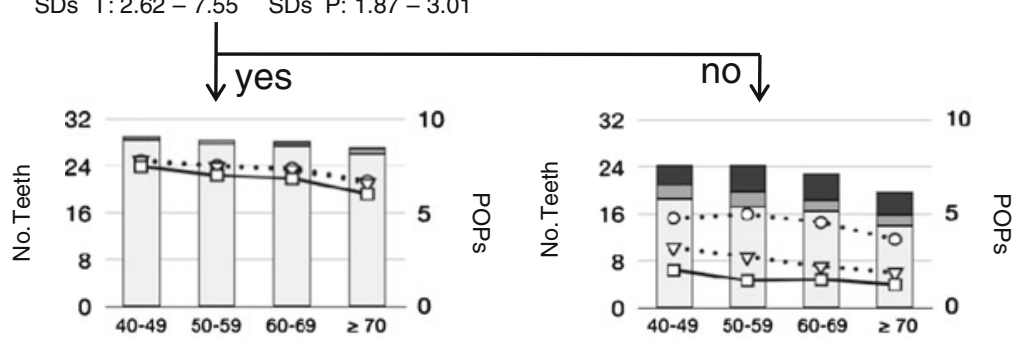

SDs T: $2.23-2.99 \quad$ SDs P: $1.64-2.16$

SDs T: $4.12-7.35$ SDs P: $1.40-2.95$

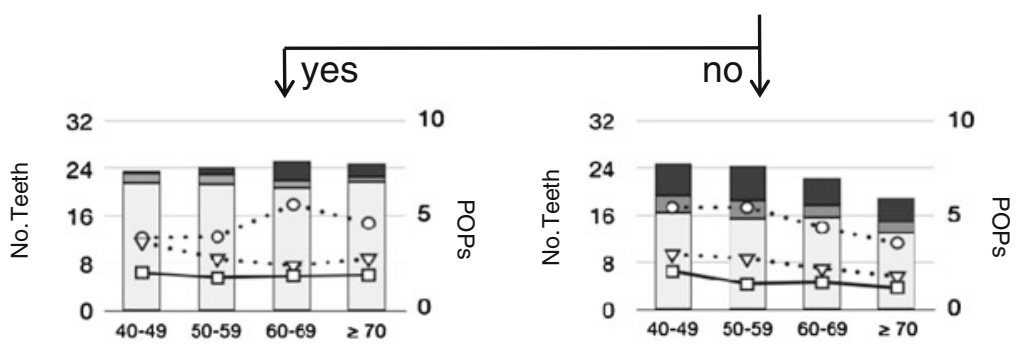

SDs T: $1.89-4.41$ SDs P: $0.99-3.28$

SDs T: $4.15-7.47$ SDs P: $1.42-2.89$

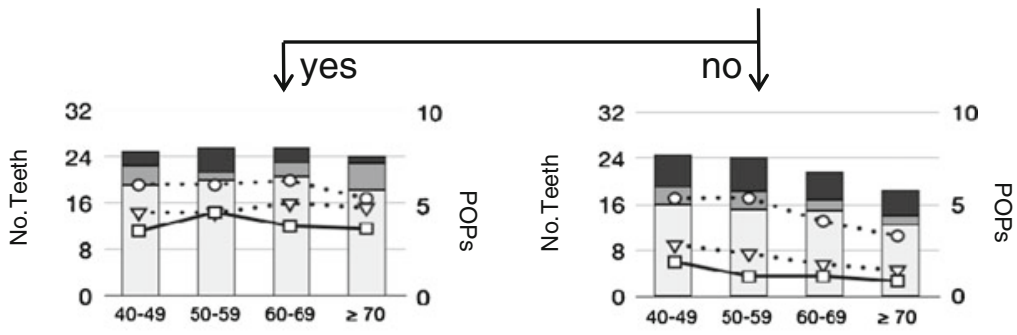

SDs $\mathrm{T}: 0.71-4.24$ SDs P: $0.44-1.86$

SDs T: $4.34-7.60$ SDs P: $1.15-2.97$
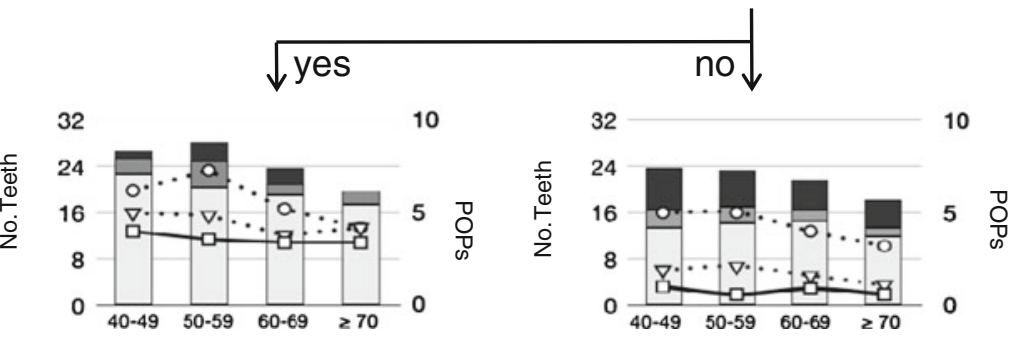

SDs $\mathrm{T}: 1.14-6.38$ SDs $\mathrm{P}: 0.55-2.55$

SDs T: $4.22-7.73$ SDs P: $0.84-3.06$

Mean number of natural teeth

- Mean number of natural posterior occluding pairs (N-POPs)

$\square$ Mean number of teeth replaced by FDPs

$-\nabla$ Mean number of natural + FDP posterior occluding pairs (N-POPs + F-POPs)

Mean number of teeth replaced by RDPs

- Mean number of natural + FDP + RDP posterior occluding pairs (N-POPs + F-POPs + R-POPs)

Fig. 3 Mean number of teeth and posterior occluding pairs per age group according to the step-by-step branching hierarchy dichotomized (yes/no) at five levels: $I \geq 1$ tooth in each jaw, $I I \geq 10$ natural teeth in each jaw, $I I I$ anterior region complete, $I V$ premolar region "sufficient," $V$ molar region "sufficient." SDs $T$ Standard deviations number of teeth; SDs $P$ standard deviations number of POPs 
of teeth replaced was 6.3 , of which $66 \%$ were replaced by RDPs (Fig. 3). In this branch, the highest increase in teeth replaced was noted for the category "impaired premolar region." With regard to the number of POPs added by replacements, the mean number of F-POPs was low for all categories in this branch. The mean number of R-POPs was relative high for subjects not meeting the criteria at the different levels, being highest in the youngest age groups with impaired molar regions (R-POPs, $3.08 \pm 2.72$ ).

Effectiveness by "promotions" in the classification system

Of the subjects having any replacement (FDP or RDP or both), 60\% (334 subjects) "promoted" to higher levels in the classification system: with FDPs, this percentage was 46; with RDPs, this was $79 \%$ (Table 4). Of the subjects that had their teeth replaced by RDPs, 79\% could be reclassified to a higher level. In the "promoted" subjects, the mean number of teeth added by FDPs was $3.53 \pm 2.43$; RDPs added on average $8.57 \pm 5.71$ teeth. In the mixed group (subjects having any replacement), the mean number of teeth added in the promoted group was $5.93 \pm 5.14$.

In subjects having teeth replaced by RDPs, promotions to higher categories occurred more frequently $(57-75 \%)$ than in subjects that had FDPs (39-46\%). The largest difference can be seen for the category of "less than ten teeth in each jaw": $75 \%$ of the subjects eligible for promotion to this category actually promoted to "at least ten teeth in each jaw" because of teeth replaced with RDP, whereas for subjects having teeth replaced by FDP, only $45 \%$ promoted.

In subjects with RDPs, also higher value promotions (mean promotion value, $5.85 \pm 3.00$ ) were found compared to subjects with FDPs (mean promotion value, $3.38 \pm 2.22$ ). However, the mean promotion value per tooth added did not differ significantly between subjects having an FDP and those having an RDP $(0.58 \pm 0.86$ and $0.72 \pm 0.68$, respectively; $t$ test, $p=0.068$ ). In contrast, for subjects that did promote to higher categories, the added value per tooth replaced was significantly higher for FDPs than for RDPs $(1.25 \pm 0.88$ and $0.92 \pm 0.65$, respectively; $t$ test, $p<0.001)$.

\section{Discussion}

This study aimed to explore the dental functional status with and without prosthodontic replacement in a Chinese adult population. As this study is part of a larger epidemiological survey, sample construction aimed at equal distribution of subjects according to residence, gender, and age. In the present report, a hierarchical classification system [8, 12], which was designed on the basis of the assumption of different qualities for different tooth types and their roles in a functional dentition, was used to

Table 4 Number of subjects (percent) eligible for promotion with fixed (FDP) and removable (RDP) dental prostheses that promoted in the dental functional classification system, mean promotion values, and mean promotion values per tooth added

\begin{tabular}{|c|c|c|c|c|c|c|}
\hline & \multicolumn{2}{|c|}{$\begin{array}{l}\text { Subjects having FDP } \\
\text { or RDP or both }\end{array}$} & \multicolumn{2}{|c|}{$\begin{array}{l}\text { Subjects } \\
\text { having FDP }\end{array}$} & \multicolumn{2}{|c|}{$\begin{array}{l}\text { Subjects } \\
\text { having RDP }\end{array}$} \\
\hline & $N(\%)$ & $\begin{array}{l}\text { Teeth added } \\
\text { mean }(\mathrm{SD})\end{array}$ & $N(\%)$ & $\begin{array}{l}\text { Teeth added } \\
\text { mean (SD) }\end{array}$ & $N(\%)$ & $\begin{array}{l}\text { Teeth addec } \\
\text { mean (SD) }\end{array}$ \\
\hline No promotion & $224(40)$ & $2.05(1.77)$ & $237(54)$ & $2.16(1.71)$ & $34(21)$ & $3.76(3.85)$ \\
\hline One or more promotions & $334(60)$ & $5.93(5.14)$ & $204(46)$ & $3.53(2.43)$ & $126(79)$ & $8.57(5.71)$ \\
\hline Promotion to functional level (value) & \multicolumn{2}{|l|}{$N(\%)^{\mathrm{a}}$} & \multicolumn{2}{|l|}{$N(\%)$} & \multicolumn{2}{|l|}{$N(\%)$} \\
\hline $\mathrm{II} \geq 10$ teeth in each jaw (4) & \multicolumn{2}{|l|}{$144(77)$} & \multicolumn{2}{|l|}{$54(45)$} & \multicolumn{2}{|l|}{$77(75)$} \\
\hline III Anterior region complete (3) & \multicolumn{2}{|l|}{$153(53)$} & \multicolumn{2}{|l|}{$82(39)$} & \multicolumn{2}{|l|}{$66(57)$} \\
\hline IV "Sufficient" premolar region (2) & \multicolumn{2}{|l|}{$167(70)$} & \multicolumn{2}{|l|}{$77(46)$} & \multicolumn{2}{|l|}{$79(73)$} \\
\hline V "Sufficient" molar region (1) & \multicolumn{2}{|l|}{$155(59)$} & \multicolumn{2}{|l|}{$74(39)$} & \multicolumn{2}{|l|}{$73(67)$} \\
\hline Mean (SD) promotion value ${ }^{\mathrm{b}}$ & \multicolumn{2}{|l|}{$4.56(2.91)$} & \multicolumn{2}{|c|}{$3.38(2.22)$} & \multicolumn{2}{|c|}{$5.85(3.00)$} \\
\hline $\begin{array}{l}\text { Mean (SD) promotion value per tooth added for subjects } \\
\text { with or without promotion }{ }^{\mathrm{b}}\end{array}$ & \multicolumn{2}{|l|}{$0.66(0.83)$} & \multicolumn{2}{|c|}{$0.58(0.86)^{*}$} & \multicolumn{2}{|c|}{$0.72(0.68)^{*}$} \\
\hline $\begin{array}{l}\text { Mean (SD) promotion value per tooth added for subjects } \\
\text { with promotion }\end{array}$ & \multicolumn{2}{|l|}{$1.11(0.81)$} & \multicolumn{2}{|c|}{$1.25(0.88)^{* *}$} & \multicolumn{2}{|c|}{$0.92(0.65)^{* *}$} \\
\hline Total number of subjects with tooth replacements & \multicolumn{2}{|l|}{558} & \multicolumn{2}{|l|}{441} & \multicolumn{2}{|l|}{160} \\
\hline
\end{tabular}

Promotion values: $1=$ promoted to "sufficient" molar region; $2=$ promoted to "sufficient" premolar region; $3=$ promoted to anterior region complete; $4=$ promoted to $\geq 10$ teeth in each jaw

${ }^{\text {a }}$ Percentage of subjects eligible for promotion to the respective functional levels

${ }^{\mathrm{b}}$ Including subjects with no promotion; promotion value $=0$

${ }^{*} p=0.068$, no significant difference; ${ }^{* *} p<0.001$, significant difference 
describe oral functional status. The homogeneities of the system were studied as a measure of its validity. The homogeneity of the dichotomized groups described in this study was comparably satisfactory as in a study performed in a Vietnamese population [8]. With respect to the number of teeth, homogeneities in the branch "at least ten teeth in each jaw," which represent $82 \%$ of all subjects with teeth in both jaws, generally were good except for the group "anterior region incomplete." In contrast, homogeneities for number of teeth in the branch of "less than ten teeth in each jaw" (18\% of subjects with teeth in both jaws) were moderate. Homogeneities with respect to POPs were good in both branches except for the categories "anterior region incomplete."

Apart from subjects edentulous in one or both jaws (8\% of the total sample), a majority $(76 \%)$ had at least ten teeth in each jaw. In this branch, given the number of teeth and POPs, most dentitions can be considered functionally "sufficient." In contrast, in the branch "less than ten teeth in each jaw" ( $16 \%$ of the total sample), most dentitions can be considered functionally "impaired."

Looking at tooth replacements, it can be seen that they appear to be made more or less following the level of functionality: in the "at least ten teeth in each jaw" branch, only few teeth were replaced (predominantly by FDPs); in the "less than ten teeth in each jaw" branch, on average, six teeth were replaced (mainly by RDPs). Generally, in the latter branch, the low numbers of natural POPs were raised by prosthetic tooth replacement up to approximately five POPs in the younger age groups and approximately four POPs in the older age groups (Fig. 2). This trend has also been reported in a systematic review on the prevalence of various types of prosthodontic restorations in Europe [13].

The classification system appeared to be able to demonstrate and to quantify effectiveness of tooth replacement in terms of upgrading dental functional status at population level. With respect to the values assigned to the promotions at different levels in this study, it can be argued that these are arbitrary. It is for instance arbitrary that promotion at level III deserves a value that is three times the value of promotion at level $\mathrm{V}$. However, the different values assigned to promotions at different levels reflect generally accepted functional importance of different tooth types and dental regions $[6,14-16]$. Although the "exact" ratio between the values for functionality is unknown, the finding of higher mean promotion values per tooth added by FDP underlines the accuracy of the values assigned.

Overall, almost $80 \%$ of RDP tooth replacement resulted in a promotion, whereas $<50 \%$ of FDP replacement resulted in a promotion. RDP replacements also resulted in a higher mean promotion value, which can be explained by the higher numbers of teeth replaced, which more often resulted in a promotion at the "at least ten teeth in each jaw" level. However, the mean promotion value per tooth added by FDPs was higher than by RDPs. This suggests that the effectiveness of tooth replacement per tooth is higher for FDP (more added value per tooth replaced). In conclusion, RDPs seem to be more effective in the classification system only when higher numbers of teeth are replaced, while FDPs are more effective per artificial tooth added.

However, the present study gives no answer to the questions whether "more added value" is reflecting true functional increase in terms of chewing ability and oral health-related quality of life. Where this study reports "more added value" per tooth added for FDPs, other studies reported better oral-health-related quality of life and better masticatory efficiency after FDP treatment compared to RDP treatment [9, 17, 18]. For chewing ability, the discerning capacity and accuracy of the classification system has previously been demonstrated in a Vietnamese population [12]. Whether this is the case for this Chinese population remains to be investigated.

On the basis of homogeneities of groups after dichotomization with the presented cut-offs, the present study demonstrated the sensibility of the classification system to display the dental functional status and the consequences of tooth replacements in this Chinese adult population.

Acknowledgment The authors appreciate the administrative support from local authorities of Zhugou and Qingdao City and are grateful for the support from Radboud University Nijmegen, The Netherlands, and the Affiliated Hospital of Medical School, Qingdao University, China.

Conflict of interest The authors declare that they have no conflict of interest.

Open Access This article is distributed under the terms of the Creative Commons Attribution Noncommercial License which permits any noncommercial use, distribution, and reproduction in any medium, provided the original author(s) and source are credited.

\section{References}

1. Luan WM, Baelum V, Chen X, Fejerskov O (1989) Dental caries in adult and elderly Chinese. J Dent Res 68:1771-1776

2. Peng B, Petersen PE, Tai BJ, Yuan BY, Fan MW (1997) Changes in oral health knowledge and behaviour 1987-95 among inhabitants of Wuhan City, PR China. Int Dent J 47:142-147

3. World Health Organization (1992) Recent advances in oral health. Report of a WHO Expert Committee. World Health Organ Tech Rep Ser 826:16-17

4. Zhang Q, Kreulen CM, Witter DJ, Creugers NHJ (2007) Oral health status and prosthodontic conditions of Chinese adults: a systematic review. Int J Prosthodont 20:567-572

5. Locker D, Slade G (1994) Association between clinical and subjective indices of oral health status in an older adult population. Gerodontology 11:108-114 
6. Gotfredsen K, Walls AWG (2007) What dentition assures oral function? Clin Oral Implant Res 18(suppl 3):34-45

7. Ikebe K, Matsuda K, Murai S, Maeda Y, Nokubi T (2010) Validation of the Eichner Index in relation to occlusal force and masticatory performance. Int J Prosthodont 23:521-524

8. Nguyen TC, Witter DJ, Bronkhorst EM, Pham LH, Creugers NHJ (2011) Dental function status in a Southern Vietnamese adult population - an analysis by a combined quantitative and qualitative classification system. Int J Prosthodont 24:30-37

9. Ueno M, Yanagisawa T, Shinada K, Ohara S, Kawaguchi Y (2010) Category of functional tooth units in relation to the number of teeth and masticatory ability in Japanese adults. Clin Oral Investig 14:113-119

10. Ueno M, Yanagisawa T, Shinada K, Ohara S, Kawaguchi Y (2008) Masticatory ability and functional tooth units in Japanese adults. J Oral Rehabil 35:337-344

11. World Health Organization (1997) Oral health surveys: basic methods, 4th edn. World Health Organization, Geneva

12. Nguyen TC, Witter DJ, Bronkhorst EM, Creugers, NHJ (2011) Chewing ability and dental functional status. Int $\mathrm{J}$ Prosthodont $24: 428-436$
13. Zitzmann NU, Hagmann E, Weiger R (2007) What is the prevalence of various types of prosthetic dental restorations in Europe? Clin Oral Implants Res 18(supp 3):20-33

14. Leake JL, Hawkins R, Locker D (1994) Social and functional impact of reduced posterior dental units in older adults. J Oral Rehabil 21:1-10

15. Rosenoer LM, Sheiham A (1995) Dental impacts on daily life and satisfaction with teeth in relation to dental status in adults. J Oral Rehabil 22:468-480

16. Elias AC, Sheiham A (1998) The relationship between satisfaction with mouth and number and position of teeth. J Oral Rehabil 25:649-661

17. Strassburger C, Kerschbaum T, Heydecke G (2006) Influence of implant and conventional prostheses on satisfaction and quality of life: a literature review. Part 2: qualitative analysis and evaluation of the studies. Int J Prosthodont 19:339-348

18. John MT, Slade GD, Szentpetery A, Setz JM (2004) Oral healthrelated quality of life in patients treated with fixed, removable, and complete dentures 1 month and 6 to 12 months after treatment. Int J Prosthodont 17:503-511 